Wilfrid Laurier University

Scholars Commons @ Laurier

2005

\title{
A General Treatment of Deformation Effects in Hamiltonians for Inhomogeneous Crystalline Materials
}

\author{
Benny Lassen \\ University of Southern Denmark \\ Morten Willatzen \\ University of Southern Denmark \\ Roderick V.N. Melnik \\ Wilfrid Laurier University, rmelnik@wlu.ca \\ L.C. Lew Yan Voon \\ Wright State University
}

Follow this and additional works at: https://scholars.wlu.ca/math_faculty

\section{Recommended Citation}

Lassen, Benny; Willatzen, Morten; Melnik, Roderick V.N.; and Yan Voon, L.C. Lew, "A General Treatment of Deformation Effects in Hamiltonians for Inhomogeneous Crystalline Materials" (2005). Mathematics Faculty Publications. 41.

https://scholars.wlu.ca/math_faculty/41

This Article is brought to you for free and open access by the Mathematics at Scholars Commons @ Laurier. It has been accepted for inclusion in Mathematics Faculty Publications by an authorized administrator of Scholars Commons@ Laurier. For more information, please contact scholarscommons@wlu.ca. 


\title{
A general treatment of deformation effects in Hamiltonians for inhomogeneous crystalline materials
}

\author{
B. Lassen and M. Willatzen \\ Mads Clausen Institute for Product Innovation, University of Southern Denmark, \\ Grundtvigs Allé 150, DK-6400 Sønderborg, Denmark \\ R. Melnik \\ Mads Clausen Institute for Product Innovation, University of Southern Denmark, \\ Grundtvigs Allé 150, DK-6400 Sønderborg, Denmark and CRC in Mathematical Modelling, \\ Wilfrid Laurier University, 75 University Avenue West, Waterloo, ON, N2L 3C5 Canada \\ L. C. Lew Yan Voon \\ Department of Physics, Wright State University, 3640 Colonel Glenn Hwy., \\ Dayton, $\mathrm{OH} 43435$
}

(Received 26 April 2005; accepted 6 September 2005; published online 9 November 2005)

In this paper, a general method of treating Hamiltonians of deformed nanoscale systems is proposed. This method is used to derive a second-order approximation both for the strong and weak formulations of the eigenvalue problem. The weak formulation is needed in order to allow deformations that have discontinuous first derivatives at interfaces between different materials. It is shown that, as long as the deformation is twice differentiable away from interfaces, the weak formulation is equivalent to the strong formulation with appropriate interface boundary conditions. It is also shown that, because the Jacobian of the deformation appears in the weak formulation, the approximations of the weak formulation is not equivalent to the approximations of the strong formulation with interface boundary conditions. The method is applied to two one-dimensional examples (a sinusoidal and a quantum-well potential) and one two-dimensional example (a freestanding quantum wire), where it is shown that the energy eigenvalues of the second-order approximations lie within $1 \%$ of the exact energy eigenvalues for a linear strain of up to $9.8 \%$, whereas the first-order approximation has an error of less than $1 \%$ for a linear strain of up to 5.5\%. (C) 2005 American Institute of Physics.

[DOI: $10.1063 / 1.2098531]$

\section{INTRODUCTION}

Nanoscale semiconductor devices, such as quantum wells, wires, and dots, usually consist of materials with different lattice constants; hence, these structures will be strained. It is known that strain strongly affects the electronic and optical properties. This has for example been used in band structure engineering of quantum-well systems; see Ref. 1 for a review including theoretical and experimental results. Also, Johnson and Bose ${ }^{2}$ have shown the necessity of including strain in the modeling of a system of coupled quantum dots, and it is known that the formation of pyramidal InAs quantum dots on GaAs substrates (self-assembled structures) is driven by the large lattice mismatch between the two semiconductors. ${ }^{3}$ Thus, in order to be able to model these devices it is important to account for strain effects. It is well known how to incorporate homogeneous strain in electronic band structure calculations, ${ }^{4}$ but in nanoscale heterostructures strain is no longer homogeneous. Zhang ${ }^{5}$ and Suzuki ${ }^{6}$ have proposed two different methods to include linear inhomogeneous strain terms in the Hamiltonian under certain assumptions for the strain and the potential. In this paper, a general method of treating Hamiltonians of deformed nanoscale systems (e.g., an inhomogeneous strained Hamiltonian) is proposed based on a Taylor series expansion. The advantage of this method is that fewer assumptions for the strain and the Hamiltonian are needed 
compared to previous models ${ }^{4-6}$ and, in addition, it is easily extended to a higher-order approximation. Finally, because the weak formulation of the problem is used, discontinuities in the gradient of the deformation are handled in an appropriate manner. Higher-order theories for Hamiltonian systems have previously been handled in the literature, e.g., in connection with Lagrangian systems for classical mechanics and field theory; see Ref. 7 and references therein.

In Sec. II, the problem is presented and the Hamiltonian appropriate for calculating the quantum-mechanical energy eigenvalues and corresponding eigenstates is specified. In Sec. II A, the mathematical tools needed to make a Taylor series expansion of the Hamiltonian with respect to a deformation of the system is presented. Section II B is concerned with the derivation of the second-order Taylor series expansion of the Hamiltonian and in Sec. II C the weak formulation of the problem is given, after which the second-order Taylor series expansion is derived for the weak formulation. Finally, in Sec. III, three examples of the application of the method are presented.

\section{THEORY}

The problem under investigation in this paper is how to model the behavior of an electron in a deformed system in general, and, more specifically, how to model the behavior of an electron in a deformed inhomogeneous crystal structure, based on the Schrödinger equation. Assuming that an undeformed domain $\mathcal{B} \subseteq \mathbb{R}^{3}$, e.g., a crystal volume, is given, a deformation $\phi: \mathcal{B} \rightarrow \mathbb{R}^{3}$ of the undeformed domain $\mathcal{B}$ is defined to be a $C^{2}$ map satisfying certain requirements to be specified in the next section. Primed ( ') coordinates, functions, and operators always refer to the domain $\mathcal{B}$ in the following, while unprimed coordinates, functions, and operators refer to the domain $\phi(\mathcal{B})$.

The deformation of a nanoscale system, as well as any material system in general, is found by minimizing the elastic energy of the system. There are basically two main approaches, an atomic approach $^{8}$ and a continuum approach. ${ }^{9-11}$ If the deformation has been found using an atomic approach, it is necessary to use the information about how the individual atoms are shifted to construct a $C^{2}$ map, but this is outside the scope of this article, i.e., it will just be assumed that the deformation has been found using a continuum approach and that this results in a $C^{2}$ map (the $C^{2}$ demand will be weakened slightly in Sec. II C).

Assuming that an electron in a potential deformed by $\phi$ is subject to the potential $\widetilde{V}_{\phi}$, the Hamiltonian of the deformed system, referred to as the deformed Hamiltonian, is given by the Schrödinger equation

$$
\widetilde{H}_{\phi}=-\frac{\hbar^{2}}{2 m} \Delta+\widetilde{V}_{\phi}
$$

It should be noted that the potential is a function of the deformed domain $\phi(\mathcal{B})$, i.e., $\widetilde{V}_{\phi}(x)$, where $x$ are coordinates on $\phi(\mathcal{B})$. The energies $E$ and corresponding wave functions $\psi$ are found by solving the eigenvalue equation

$$
\widetilde{H}_{\phi} \psi=E \psi
$$

subject to certain boundary conditions. For an electron in a crystal, the most important potential contributions are

$$
\tilde{V}_{\phi}=V_{\phi}+\hat{H}^{\mathrm{SO}}=V_{\phi}-i \frac{\hbar^{2}}{2 m^{2} c^{2}}\left(\nabla V_{\phi} \times \nabla\right) \cdot \sigma,
$$

where $V_{\phi}$ is the potential of the deformed crystal without spin, $\hat{H}^{\mathrm{SO}}$ is the spin-orbit part, and $\sigma$ are the Pauli spin matrices. This is the situation considered in this work, but it should be noted that the procedure can be used on any potential which is a differential function of $\phi$ in a sense to be defined later. In order to incorporate spin, operators on the function space $\mathcal{F}$ are extended to $\mathcal{F}$ $\times \mathcal{F}$ by operating on each element of the pair separately, e.g., let $f \in \mathcal{F} \times \mathcal{F}$, i.e., $f=\left(\begin{array}{l}f_{+} \\ f_{-}\end{array}\right)$, where $f_{+}, f_{-} \in \mathcal{F}$; then 


$$
V_{\phi} f=V_{\phi}\left(\begin{array}{c}
f_{+} \\
f_{-}
\end{array}\right)=\left(\begin{array}{c}
V_{\phi} f_{+} \\
V_{\phi} f_{-}
\end{array}\right) .
$$

The function $f_{+}$corresponds to the spin-up state and $f_{-}$corresponds to the spin-down state.

The potential of the undeformed domain is usually better known than the potential of the deformed domain. Assuming that the deformation is known, it is therefore advantageous to express the deformed Hamiltonian with respect to the deformation and the undeformed potential. This can be achieved by making a Taylor series expansion of the deformed Hamiltonian with respect to the deformation. In order to do so, the deformed Hamiltonian is written with respect to coordinates on $\mathcal{B}$. This can be done as long as the deformation is invertible with twice differentiable inverse. The deformed Hamiltonian is, in that case, given by

$$
\tilde{H}_{\phi}=-\frac{\hbar^{2}}{2 m} \Delta+V_{\phi}-i \frac{\hbar^{2}}{2 m^{2} c^{2}}\left(\nabla V_{\phi} \times \nabla\right) \cdot \sigma .
$$

Changing to coordinates on $\mathcal{B}$, the deformed Hamiltonian takes the form

$$
H_{\phi}=K_{F}+V_{\phi}{ }^{\circ} \phi+H_{\phi}^{\mathrm{SO}},
$$

where $F=\nabla^{\prime} \phi$ and the kinetic and spin-orbit parts $\left(K_{F}\right.$ and $H_{\phi}^{\mathrm{SO}}$, respectively) are given by

$$
K_{F}=-\frac{\hbar^{2}}{2 m}\left[F^{-1}\left(\phi\left(\mathbf{r}^{\prime}\right)\right)\right]_{j i} \frac{\partial}{\partial x_{j}^{\prime}}\left(\left[F^{-1}\left(\phi\left(\mathbf{r}^{\prime}\right)\right)\right]_{k i} \frac{\partial}{\partial x_{k}^{\prime}}\right),
$$

and

$$
H_{\phi}^{\mathrm{SO}}=-i \frac{\hbar^{2}}{4 m^{2} c^{2}}\left(\left[F^{-1}\left(\phi\left(\mathbf{r}^{\prime}\right)\right)\right]^{T} \nabla^{\prime} V_{\phi}\left(\phi\left(\mathbf{r}^{\prime}\right)\right) \times\left[F^{-1}\left(\phi\left(\mathbf{r}^{\prime}\right)\right)\right]^{T} \nabla^{\prime}\right) \cdot \sigma .
$$

Here and throughout the paper, Einstein's summation convention is applied, i.e., summation over repeated indices, e.g., $a_{i} b_{i}=\sum_{i=1}^{3} a_{1} b_{i}$. It should be noted that $\widetilde{H}_{\phi}$ in Eq. (2) and $H_{\phi}$ in Eq. (3) are related by a unitary transformation (change of coordinates). It is possible to use other unitary transformations to write the Hamiltonian with respect to coordinates on $\mathcal{B}$, e.g., a transformation similar to the one used in Hislop et al. ${ }^{12}$ in connection with spectral deformation theory, which take into account the determinant of the deformation gradient appearing in the probability distribution by solving for $\sqrt{\operatorname{det} F} \psi(\mathbf{r})$ instead of $\psi(\mathbf{r})$. The Taylor series expansion will of course reflect the choice of transformation, but the order of the approximation will stay the same.

The idea now is to make a Taylor series expansion of $H_{\phi}$ [Eq. (3)] with respect to the deformation $\phi$. Hence, it is necessary to set up the mathematical theory required to handle the above situation. This will be done in the next section.

\section{A. Mathematical tools}

The first thing needed in order to use a Taylor series expansion is to define a derivative applicable in the given situation. The weakest notion of a derivative is the Gâteaux derivative, and this will be used in the present work. Let $U$ and $V$ be normed linear spaces, $S \subseteq U$ an open set, and $P$ an operator from $S$ to $V$. In addition, let $u_{0} \in S, \eta \in U$ nonzero, and $\mathcal{I}=(-\alpha, \alpha)$, where $\alpha \in \mathbb{R}$ is chosen such that $u_{0}+t \eta \in U$ for all $t \in \mathcal{I}$. The Gâteaux differential of $P$ at $u_{0}$ in the direction $\eta$ is then defined to be

$$
D P\left(u_{0}\right) \cdot \eta \equiv \frac{d}{d t}\left(P\left(u_{0}+t \eta\right)\right)=\lim _{t \rightarrow 0} \frac{1}{t}\left(P\left(u_{0}+t \eta\right)-P\left(u_{0}\right)\right),
$$

when the limit exists. An operator $P$ which has a Gâteaux differential at each point in $S$ in any direction is said to have a Gâteaux differential on $S .{ }^{13}$ The higher-order differentials are introduced inductively in the usual manner. It should be noted that when the Gâteaux derivative fulfills certain 
additional requirements it is equal to the stronger, and more widely used, concept of a derivative called Frechet differentiability. ${ }^{14}$ This concept has, among other things, the advantage of being linear in $\eta$, which is not always the case for the Gâteaux derivative.

The version of Taylor's theorem that is relevant for this work is given as follows.

Theorem II.1. Let $U$ and $V$ be normed linear spaces. Suppose that the space $V$ is complete and that the region $S$ of the space $U$ is convex, and suppose that the function $P$ from $S$ to $V$ has an nth Gâteaux differential on $S$. Suppose also that for every $u_{1}, u_{2}$ in $S$ the function of $r$, $D^{n} P\left(u_{1}+\left(u_{2}-u_{1}\right) r\right) \cdot\left(u_{2}-u_{1}\right)^{n}$, is bounded on the interval $(0,1)$ and its set of discontinuities is of measure zero. Then, for every $u_{1}, u_{2}$ in $S$ we have

$$
P\left(u_{2}\right)=P\left(u_{1}\right)+\sum_{i=1}^{n-1} \frac{D^{i} P\left(u_{1}\right) \cdot\left(u_{2}-u_{1}\right)^{i}}{i !}+R_{n}\left(u_{1}, u_{2}\right),
$$

where

$$
R_{n}\left(u_{1}, u_{2}\right)=\int_{0}^{1} D^{n} P\left(u_{1}+\left(u_{2}-u_{1}\right) r\right) \cdot\left(u_{2}-u_{1}\right)^{n} \frac{(1-r)^{n-1}}{(n-1) !} d r .
$$

The statement that a subset of an interval is of measure zero is equivalent to the statement that the total length of the subset is zero. For a proof of this theorem and a definition of the Riemann integral appearing in the theorem, refer to Ref. 13. In order to see how this theorem can be used in the situation given above, a more precise definition of a deformation, and of the corresponding deformed Hamiltonian, is necessary. This will be given in the following.

First, the overall normed linear space that a deformation belongs to is defined. Let $f$ be a once differentiable function from $\mathcal{B}$ to $\mathbb{R}^{3}$, and define

$$
\|f\|_{C^{1}}=\left\{\sup _{\mathcal{B}}\left[f_{1}^{2}+\sum_{i=1}^{3}\left(\frac{\partial f_{1}}{\partial x_{i}}\right)^{2}+f_{2}^{2}+\sum_{i=1}^{3}\left(\frac{\partial f_{2}}{\partial x_{i}}\right)^{2}+f_{3}^{2}+\sum_{i=1}^{3}\left(\frac{\partial f_{3}}{\partial x_{i}}\right)^{2}\right]\right\}^{1 / 2},
$$

where $f=\left(f_{1}, f_{2}, f_{3}\right)$. Denote with $\hat{C}^{2}$ the normed linear space consisting of all the twice differentiable functions from $\mathcal{B}$ to $R^{3}$ that have a finite norm given by (7). A deformation $\phi: \mathcal{B} \rightarrow R^{3}$ of the undeformed domain $\mathcal{B}$ is defined to be an injective $\hat{C}^{2}$ map satisfying $\inf \left(\operatorname{det}\left(\nabla^{\prime} \phi\right)\right)>0$. The last requirement is needed to ensure that the map has a twice differentiable inverse; this is guaranteed by the inverse function theorem. The existence of the inverse ensures that the deformation does not destroy parts of the domain, e.g., by collapsing or tearing parts of the domain. It is assumed that $\mathcal{B}$ is bounded; this ensures boundedness of considered operators. The space of all deformations of $\mathcal{B}$ is denoted $\mathcal{D}$. It can be shown that the function $g: \hat{C}^{2} \rightarrow \mathbb{R}$ given by $g(\phi)$ $=\inf \left(\operatorname{det}\left(\nabla^{\prime} \phi\right)\right)$ is continuous, and $\mathcal{D}$ is, as a consequence, open in $\hat{C}^{2}$ (it is the inverse image of the open set $] \infty, 0[$ ).

Next, the deformed Hamiltonian is shown to be a function from $\mathcal{D}$ to a normed linear space. The domain of the deformed Hamiltonian is, in this article, chosen to be the product of Sobolev spaces: $H^{2}(\phi(\mathcal{B})) \times H^{2}(\phi(\mathcal{B}))$. This is the largest space for which the deformed Hamiltonian is defined. ${ }^{15}$ The deformation has, according to the inverse function theorem, a twice differentiable inverse. This ensures that $H_{\phi}$ can be written with respect to coordinates on $\mathcal{B}$, as it was done in Eq. (3). As a consequence, the deformed Hamiltonian can be viewed as an operator

$$
\hat{H}: \mathcal{D} \rightarrow L\left(H^{2}(\mathcal{B}) \times H^{2}(\mathcal{B}), \quad L^{2}(\mathcal{B}) \times L^{2}(\mathcal{B})\right),
$$

given by $\phi \mapsto H_{\phi}$, where $L(\mathcal{F}, \mathcal{G})$ is the space of bounded linear mappings from $\mathcal{F}$ to $\mathcal{G}$ and $L^{2}(\mathcal{B})$ is the Lebesgue space. The Lebesgue space is needed here because of the definition of the Sobolev space. ${ }^{15}$ It is also the appropriate space with respect to wave functions, because these give the probability that an electron is in a certain area, and, as a consequence, they only need to be defined 
almost everywhere, i.e., up to sets of zero measure. The spaces $H^{2}(\mathcal{B}) \times H^{2}(\mathcal{B})$ and $L^{2}(\mathcal{B})$ $\times L^{2}(\mathcal{B})$ are equipped with the $L^{2}$ norm

$$
\|\psi\|_{L^{2}}=\left(\int_{\mathcal{B}} \psi_{+}^{*} \psi_{+} \mathrm{d} V\right)^{1 / 2}+\left(\int_{\mathcal{B}}\left(\psi_{-}^{*} \psi_{-}\right) \mathrm{d} V\right)^{1 / 2}
$$

where $\psi=\left(\begin{array}{c}\psi_{+} \\ \psi_{-}\end{array}\right)$and $\psi_{+}, \psi_{-}$are functions from $\mathcal{B}$ to $\mathrm{C}$. The space $L^{2}(\mathcal{B}) \times L^{2}(\mathcal{B})$ equipped with this norm is a complete normed linear space. The space $L\left(H^{2}(\mathcal{B}) \times H^{2}(\mathcal{B}), L^{2}(\mathcal{B}) \times L^{2}(\mathcal{B})\right)$ is equipped with the operator norm

$$
\|A\|_{O}=\sup \left\{\|A \psi\|_{L^{2}} \mid \psi \in H^{2}(\mathcal{B}) \times H^{2}(\mathcal{B}), \quad\|\psi\|_{L^{2}} \leqslant 1\right\},
$$

where $A \in L\left(H^{2}(\mathcal{B}) \times H^{2}(\mathcal{B}), L^{2}(\mathcal{B}) \times L^{2}(\mathcal{B})\right)$. This makes the space $L\left(H^{2}(\mathcal{B}) \times H^{2}(\mathcal{B}), L^{2}(\mathcal{B})\right.$ $\times L^{2}(\mathcal{B})$ ) into a complete normed linear space (see Theorem III.2.3 in Ref. 16).

The function to be approximated by a Taylor series expansion is the function $\hat{H}$, i.e., with respect to Theorem II.1: $P=\hat{H}, U=\hat{C}^{2}$, and $V=L\left(H^{2}(\mathcal{B}) \times H^{2}(\mathcal{B}), L^{2}(\mathcal{B}) \times L^{2}(\mathcal{B})\right)$. Depending on which element of $\mathcal{D}$ the deformed Hamiltonian is expanded about, the convexity requirement can be fulfilled by restricting the domain of $\hat{H}$ to the largest open ball with respect to the norm ${ }^{7}$ that contains the element of expansion and is contained in $\mathcal{D}$. This restriction normally poses no problems because Taylor series expansions are in general only accurate within small deviations from the element of expansion. The restricted domain is the set $S$ in Theorem II.1. That the Gâteaux derivatives exists and that $D^{n} P\left(u_{1}+\left(u_{2}-u_{1}\right) r\right) \cdot\left(u_{2}-u_{1}\right)^{n}$ fulfills the given requirements has to be verified for each specific potential $V_{\phi}$.

\section{B. Second-order deformed Hamiltonian}

In this section, the second-order Taylor series expansion of the deformed Hamiltonian around the identity map id on $\mathcal{B}$ is presented. In order to find the second-order Taylor series expansion, it is necessary to find the first- and second-order Gâteaux derivatives. This is done in Appendix A. It is assumed that $V_{\phi}$ is well enough behaved such that Theorem II.1 can be used. The Taylor series expansion of the deformed Hamiltonian to the second order in $\phi$ around $i d$ is then given by

$$
H_{\phi} \approx H_{i d}+D^{(1)}(\phi-i d)+D_{\mathrm{SO}}^{(1)}(\phi-i d)+D^{(2)}(\phi-i d, \phi-i d)+D_{\mathrm{SO}}^{(2)}(\phi-i d, \phi-i d),
$$

where

$$
\begin{gathered}
H_{i d}=-\frac{\hbar^{2}}{2 m} \nabla^{\prime 2}+V_{i d}-i \frac{\hbar^{2}}{4 m^{2} c^{2}}\left(\nabla^{\prime} V_{i d} \times \nabla^{\prime}\right) \cdot \sigma, \\
D^{(1)}(\phi-i d)=\frac{\hbar^{2}}{2 m}\left\{\left[F+F^{T}-2 I\right]_{j k} \frac{\partial}{\partial x_{j}^{\prime}} \frac{\partial}{\partial x_{k}^{\prime}}+\frac{\partial}{\partial x_{i}^{\prime}}\left([F-I]_{k i}\right) \frac{\partial}{\partial x_{k}^{\prime}}\right\}+D V_{i d} \cdot(\phi-i d), \\
D_{\mathrm{SO}}^{(1)}(\phi-i d)=-i \frac{\hbar^{2}}{4 m^{2} c^{2}}\left\{\left(\nabla^{\prime}\left(D V_{i d} \cdot(\phi-i d)\right) \times \nabla^{\prime}\right) \cdot \sigma-\left((F-I)^{T} \nabla^{\prime} V_{i d} \times \nabla^{\prime}\right) \cdot \sigma\right. \\
\left.-\left(\nabla^{\prime} V_{i d} \times(F-I)^{T} \nabla^{\prime}\right) \cdot \sigma\right\},
\end{gathered}
$$




$$
\begin{aligned}
D^{(2)}(\phi-i d, \phi-i d)= & -\frac{\hbar^{2}}{2 m}\left\{\left[(F-I)(F-I)^{T}+(F-I)^{2}+\left(F^{T}-I\right)^{2}\right]_{j k} \frac{\partial}{\partial x_{j}^{\prime}} \frac{\partial}{\partial x_{k}^{\prime}}\right. \\
& \left.+[(F-I)]_{j i} \frac{\partial}{\partial x_{j}^{\prime}}\left([F-I]_{k i}\right) \frac{\partial}{\partial x_{k}^{\prime}}+\frac{\partial}{\partial x_{i}^{\prime}}\left[(F-I)^{2}\right]_{k i} \frac{\partial}{\partial x_{k}^{\prime}}\right\} \\
& +\frac{1}{2} D^{2} V_{i d} \cdot((\phi-i d),(\phi-i d)), \\
D_{\mathrm{SO}}^{(2)}(\phi-i d, \phi-i d)= & -i \frac{\hbar^{2}}{4 m^{2} c^{2}}\left\{-\left((F-I)^{T} \nabla^{\prime}\left(D V_{i d} \cdot(\phi-i d)\right) \times \nabla^{\prime}\right) \cdot \sigma\right. \\
& -\left(\nabla^{\prime}\left(D V_{i d} \cdot(\phi-i d)\right) \times(F-I)^{T} \nabla^{\prime}\right) \cdot \sigma \\
& +\left((F-I)^{T} \nabla^{\prime} V_{i d} \times(F-I)^{T} \nabla^{\prime}\right) \cdot \sigma \\
& +\frac{1}{2}\left(\nabla^{\prime}\left(D^{2} V_{i d} \cdot((\phi-i d),(\phi-i d))\right) \times \nabla^{\prime}\right) \cdot \sigma \\
& \left.+\left(\left(F^{T}-I\right)^{2} \nabla^{\prime} V_{i d} \times \nabla^{\prime}\right) \cdot \sigma+\left(\nabla^{\prime} V_{i d} \times\left(F^{T}-I\right)^{2} \nabla^{\prime}\right) \cdot \sigma\right\},
\end{aligned}
$$

and $F=\nabla^{\prime} \phi$.

To connect these expressions with the strain tensor, note that the Green-Lagrange-St. Venant strain tensor is given by

$$
\epsilon=\frac{1}{2}\left(F^{T} F-I\right)
$$

and the linear strain tensor is given by

$$
\epsilon^{\operatorname{lin}}=\frac{1}{2}\left(F+F^{T}-2 I\right)
$$

In the case where $F$ is symmetric, Eq. (9) becomes

$$
\epsilon^{\text {lin }}=F-I \text {. }
$$

If only the first-order terms of the Taylor series expansion are used, and if the linear strain is used with a symmetric deformation gradient, then the herein derived first-order approximation to the deformed Hamiltonian is identical to the one proposed by Zhang. ${ }^{5}$ In the case where the deformation is homogeneous, the first-order approximation is the same as the one derived by Bir et al. ${ }^{4}$

\section{Weak formulation}

The preceding sections are all concerned with deformations that are twice differentiable, but there are many situations where the deformations do not satisfy this. For example, in the quantumwell case the deformation is piecewise linear with different slopes inside and outside the well. ${ }^{17}$ Therefore, to be able to handle this kind of deformations, an extension of the theory to a larger class of deformations is needed.

Let $\mathcal{A}$ be the set of injective functions from $\mathcal{B}$ to $\mathbb{R}^{3}$ that are once differentiable on $\mathcal{B} \backslash \Gamma$ with both a finite $C^{1}$ norm [Eq. (7)] and a positive Jacobian determinant, where $\Gamma$ is a finite set of piecewise smooth surfaces (two-dimensional manifolds). This set of functions includes the deformations considered in quantum-well, as well as quantum-wire and quantum-dot structures (according to continuum mechanical models ${ }^{9-11}$ ). The problem with the functions belonging to $\mathcal{A}$ is that they are not necessarily once differentiable on $\mathcal{B}$ because the first derivative can have discon- 
tinuities at interfaces. Therefore, caution is needed when "pulling back" the deformed Hamiltonian to coordinates on $\mathcal{B}$ [Eq. (3)]. The correct formalism to use in this case is the weak formalism, i.e., instead of solving the eigenvalue equation

$$
H_{\phi} \tilde{\psi}=E \tilde{\psi}
$$

subject to some boundary conditions (in this work either Dirichlet or periodic boundary conditions), a solution to the weak equation

$$
\int_{\phi(\mathcal{B})} \xi_{+}^{*}\left(H_{\phi} \tilde{\psi}\right)_{+} \mathrm{d} V+\int_{\phi(\mathcal{B})} \xi_{-}^{*}\left(H_{\phi} \tilde{\psi}\right)_{-} \mathrm{d} V=\int_{\phi(\mathcal{B})} \xi_{+}^{*} E \tilde{\psi}_{+} \mathrm{d} V+\int_{\phi(\mathcal{B})} \xi_{-}^{*} E \tilde{\psi} \tilde{\psi}_{-} \mathrm{d} V,
$$

for all $\xi_{+}, \xi_{-} \in C_{c}^{\infty}(\phi(\mathcal{B}), C$ ) (smooth functions with compact support; see, e.g., Ref. 16) is found, where $\left(H_{\phi} \widetilde{\psi}\right)_{+}$is the first factor of $H_{\phi} \widetilde{\psi}$ and $\left(H_{\phi} \widetilde{\psi}\right)_{-}$is the last factor. The solution still has to satisfy the boundary conditions. Introducing

$$
\begin{gathered}
K_{\phi}^{ \pm}\left(\eta_{ \pm}, \zeta\right)=\frac{\hbar^{2}}{2 m} \int_{\mathcal{B} \backslash \Gamma}\left[\left(F^{-1} \circ \phi\right)\left(F^{-1} \circ \phi\right)^{T}\right]_{i j} \frac{\partial\left(\eta_{ \pm}\right)^{*}}{\partial x_{i}^{\prime}} \frac{\partial \zeta_{ \pm}}{\partial x_{j}^{\prime}} \operatorname{det} F \mathrm{~d} V^{\prime} \\
V_{\phi}^{ \pm}\left(\eta_{ \pm}, \zeta\right)=\int_{\mathcal{B}} \eta_{ \pm}^{*} V_{\phi}^{\circ} \phi \zeta_{ \pm} \operatorname{det} F \mathrm{~d} V^{\prime} \\
H_{\phi}^{\mathrm{SO}, \pm}\left(\eta_{ \pm}, \zeta\right)=-i \frac{\hbar^{2}}{4 m^{2} c^{2}} \int_{\mathcal{B} \backslash \Gamma} \eta_{ \pm}^{*}\left(F^{T} \sigma \times \nabla^{\prime} V_{\phi} \circ \phi \cdot \nabla^{\prime} \zeta\right)_{ \pm} \mathrm{d} V^{\prime} \\
W_{\phi}(\eta, \zeta ; E)=K_{\phi}^{+}\left(\eta_{ \pm}, \zeta\right)+V_{\phi}^{+}\left(\eta_{ \pm}, \zeta\right)+H^{\mathrm{SO},+}{ }_{\phi}\left(\eta_{ \pm}, \zeta\right)-\int_{\mathcal{B}} \eta_{+}^{*} E \zeta_{+} \operatorname{det} F \mathrm{~d} V^{\prime}+K_{\phi}^{-}\left(\eta_{ \pm}, \zeta\right)+V_{\phi}^{-}\left(\eta_{ \pm}, \zeta\right) \\
+H^{\mathrm{SO},-}{ }_{\phi}\left(\eta_{ \pm}, \zeta\right)-\int_{\mathcal{B}} \eta_{-}^{*} E \zeta_{-} \operatorname{det} F \mathrm{~d} V^{\prime},
\end{gathered}
$$

where $\eta=\left(\begin{array}{c}\eta_{+} \\ \eta_{-}\end{array}\right), \zeta=\left(\begin{array}{c}\zeta_{+} \\ \zeta_{-}\end{array}\right), \eta_{+}, \eta_{-} \in H^{2}(\mathcal{B})$, and $\zeta_{+}{ }^{\circ} \phi^{-1}, \zeta_{-}{ }^{\circ} \phi^{-1} \in C_{c}^{\infty}(\phi(\mathcal{B}), \mathbb{C})$, the weak formulation [Eq. (10)] can be put in the form: Find $\widetilde{\psi}$ and $E$ such that

$$
W_{\phi}(\xi \circ \phi, \tilde{\psi} \circ \phi ; E)=0,
$$

for all $\xi_{+}, \xi_{-} \in C_{c}^{\infty}(\phi(\mathcal{B}), C)$. The expression for the kinetic part [Eq. (11)] is found by integrating by parts, using that $\xi_{+}$and $\xi_{-}$have compact support, changing coordinates, and using the chain rule. The integral is restricted to $\mathcal{B} \backslash \Gamma$ for the chain rule to apply. This is possible because $\Gamma$ has measure zero (zero volume). Finding the expression for the potential part [Eq. (12)] is straightforward, and the expression for the spin-orbit part [Eq. (13)] is found again by changing coordinates and using the chain rule; in addition, the rule that $A v_{1} \times A v_{2} \cdot A v_{3}=\operatorname{det}(A) v_{1} \times v_{2} \cdot v_{3}$, for a $3 \times 3$ matrix $A$ and $v_{1}, v_{2}, v_{3} \in \mathbb{R}^{3}$ is used.

According to Theorem B.1 in the Appendix, the above weak formulation is equivalent to finding $\psi$ and $E$ such that

$$
W_{\phi}(\eta, \psi ; E)=0
$$

for all $\eta_{+}, \eta_{-} \in C_{c}^{\infty}\left(\mathcal{B}, C_{)}\right)$, in which case $\tilde{\psi}=\psi^{\circ} \phi^{-1}$. Equation (15) is a weak formulation of the problem with respect to the domain $\mathcal{B}$.

The operator $W$ defined in Eq. (14) can be viewed as an operator $W: \mathcal{A} \rightarrow L\left(H^{2}(\mathcal{B})\right.$ $\left.\times H^{2}(\mathcal{B}), C_{c}^{\infty}(\mathcal{B}, \mathrm{C}) \times C_{c}^{\infty}(\mathcal{B}, \mathrm{C}) ; A(\mathbb{R}, \mathbb{R})\right)$, where $L\left(\mathcal{F}_{1}, \mathcal{F}_{2} ; \mathcal{G}\right)$ is the space of bilinear mappings 
from $\mathcal{F}_{1} \times \mathcal{F}_{2}$ to $\mathcal{G}$ and $A(\mathbb{R}, \mathbb{R})$ is the space of affine mappings from $\mathbb{R}$ to $\mathbb{R}$. The space $A(\mathbb{R}, \mathbb{R})$ is a finite-dimensional normed vector space, and because of this it is complete. It follows that the range of $W$ is complete so, according to Theorem II.1, the Taylor series expansion of $W$ exists, assuming that the Gâteaux derivative exists and fulfills given requirements. The domain of $W$ can be restricted to an open convex set using similar arguments as in Sec. II A.

In Appendix B the derivatives needed to write out the second-order Taylor series expansion of $W$ are found, giving

$$
W_{\phi} \approx W_{i d}+W^{(1)}(\phi-i d)+W_{\mathrm{SO}}^{(1)}(\phi-i d)+W^{(2)}(\phi-i d, \phi-i d)+W_{\mathrm{SO}}^{(2)}(\phi-i d, \phi-i d),
$$

where

$$
\begin{aligned}
& W^{(1)}(\phi-i d)(\eta, \psi ; E)=\sum_{s=+,-}\left\{-\frac{\hbar^{2}}{2 m} \int_{\mathcal{B} \backslash \Gamma}\left[F+F^{T}-2 I\right]_{i j} \frac{\partial\left(\eta_{s}\right)^{*}}{\partial x_{i}^{\prime}} \frac{\partial \psi_{s}}{\partial x_{j}^{\prime}}-\frac{\partial\left(\eta_{s}\right)^{*}}{\partial x_{i}^{\prime}} \frac{\partial \psi_{s}}{\partial x_{i}^{\prime}} \operatorname{Tr}(F-I) \mathrm{d} V^{\prime}\right. \\
& \left.+\int_{\mathcal{B}} \eta_{s}^{*}\left(D V_{i d} \cdot(\phi-i d)+\left(V_{i d}-E\right) \operatorname{Tr}(F-I)\right) \psi_{s} \mathrm{~d} V^{\prime}\right\} \\
& W^{(2)}(\phi-i d, \phi-i d)(\eta, \psi ; E)=\sum_{s=+,-}\left\{\frac{\hbar^{2}}{2 m} \int_{\mathcal{B} \backslash \Gamma}\left[(F-I)^{2}+\left(F^{T}-I\right)^{2}+(F-I)(F-I)^{T}\right]_{i j} \frac{\partial\left(\eta_{s}\right)^{*}}{\partial x_{i}^{\prime}} \frac{\partial \psi_{s}}{\partial x_{j}^{\prime}} \mathrm{d} V^{\prime}\right. \\
& -\frac{\hbar^{2}}{2 m} \int_{\mathcal{B} \backslash \Gamma}\left[F+F^{T}-2 I\right]_{i j} \operatorname{Tr}(F-I) \frac{\partial\left(\eta_{s}\right)^{*}}{\partial x_{i}^{\prime}} \frac{\partial \psi_{s}}{\partial x_{j}^{\prime}} \mathrm{d} V^{\prime} \\
& +\frac{\hbar^{2}}{2 m} \int_{\mathcal{B} \backslash \Gamma} \frac{\partial\left(\eta_{s}\right)^{*}}{\partial x_{i}^{\prime}} \frac{\partial \psi_{s}}{\partial x_{i}^{\prime}} \operatorname{Tr}\left([F-I]_{c o}\right) \mathrm{d} V^{\prime} \\
& +\frac{1}{2} \int_{\mathcal{B}} \eta_{s}^{*} D^{2} V_{i d} \cdot(\phi-i d, \phi-i d) \psi_{s} \mathrm{~d} V \\
& \left.+\int_{\mathcal{B}} \eta_{s}^{*}\left(D V_{i d} \cdot(\phi-i d) \operatorname{Tr}(F-I)+\left(V_{i d}-E\right) \operatorname{Tr}\left([F-I]_{c o}\right)\right) \psi_{s} \mathrm{~d} V^{\prime}\right\}, \\
& W_{\mathrm{SO}}^{(1)}(\phi-i d)(\eta, \psi)=\sum_{s=+,-}-i \frac{\hbar^{2}}{4 m^{2} c^{2}} \int_{\mathcal{B} \backslash \Gamma}\left\{\eta_{s}^{*}\left((F-I)^{T} \sigma \times \nabla^{\prime} V_{i d} \cdot \nabla^{\prime} \psi\right)_{s}\right. \\
& \left.+\eta_{s}^{*}\left(\sigma \times \nabla^{\prime}\left(D V_{i d} \cdot(\phi-i d)\right) \cdot \nabla^{\prime} \psi\right)_{s}\right\} \mathrm{d} V^{\prime},
\end{aligned}
$$

and

$$
\begin{aligned}
W_{\mathrm{SO}}^{(2)}(\phi-i d, \phi-i d)(\eta, \psi)= & \sum_{s=+,-}\left\{-i \frac{\hbar^{2}}{4 m^{2} c^{2}} \int_{\mathcal{B} \backslash \Gamma} \eta_{s}^{*}\left((F-I)^{T} \sigma \times \nabla^{\prime}\left(D V_{i d} \cdot(\phi-i d)\right) \cdot \nabla^{\prime} \psi\right)_{s} \mathrm{~d} V^{\prime}\right. \\
& \left.-i \frac{\hbar^{2}}{4 m^{2} c^{2}} \int_{\mathcal{B} \backslash \Gamma} \eta_{s}^{*}\left(\sigma \times \nabla^{\prime}\left(\frac{1}{2} D^{2} V_{i d} \cdot(\phi-i d, \phi-i d)\right) \cdot \nabla^{\prime} \psi\right) \mathrm{d} V^{\prime}\right\} .
\end{aligned}
$$

In contrast to the previous deformed Hamiltonian models by Bir and Pikus, ${ }^{4}$ Zhang, ${ }^{5}$ and Suzuki, ${ }^{6}$ the present model describes a general rigoristic mathematical model for incorporating nonsmooth (and of course, smooth) deformation fields in Hamiltonians. 


\section{Strong formulation with interface boundary conditions}

The weak form can, as long as the deformations lie in $\mathcal{A}$ and are twice differentiable on $\mathcal{B} \backslash \Gamma$, be reformulated in a strong form with interface boundary conditions. This is done using integration by parts in the kinetic term [Eq. (11)]. Let $\mathbf{n}=\left(n_{1}, n_{2}, n_{3}\right)$ be a unit normal vector field on the interface $\Gamma$; then

$$
\begin{aligned}
K_{\phi}^{ \pm}\left(\eta_{ \pm}, \zeta\right)= & \frac{\hbar^{2}}{2 m} \int_{\Gamma} n_{i}\left\{\left(\left[\left(F^{-1} \circ \phi\right)\left(F^{-1} \circ \phi\right)^{T}\right]_{i j}\left(\eta_{ \pm}\right)^{*} \frac{\partial \zeta_{ \pm}}{\partial x_{j}^{\prime}} \operatorname{det} F\right)_{m 1}\right. \\
& \left.-\left(\left[\left(F^{-1} \circ \phi\right)\left(F^{-1} \circ \phi\right)^{T}\right]_{i j}\left(\eta_{ \pm}\right)^{*} \frac{\partial \zeta_{ \pm}}{\partial x_{j}^{\prime}} \operatorname{det} F\right)_{m 2}\right\} \mathrm{d} A^{\prime} \\
& -\frac{\hbar^{2}}{2 m} \int_{\mathcal{B} \backslash \Gamma}\left(\eta_{ \pm}\right)^{*}\left[F^{-1} \circ \phi\right]_{j i} \frac{\partial}{\partial x_{j}^{\prime}}\left(\left[F^{-1} \circ \phi\right]_{k i} \frac{\partial \zeta_{ \pm}}{\partial x_{k}^{\prime}}\right) \operatorname{det} F \mathrm{~d} V^{\prime}
\end{aligned}
$$

where the subscripts $m 1$ and $m 2$ symbolize that the expression in brackets is found as the limit taken from the region that the normal vector points out of and into, respectively. To get this result, it has also been used that

$$
\frac{\partial}{\partial x_{i}}\left(F_{i k}^{-1} \operatorname{det} F\right)=\frac{\partial}{\partial x_{i}}\left(\left[F_{c o}\right]_{k i}\right)=0
$$

for $k=1,2,3$, where $F_{c o}$ is the matrix of cofactors of $F$. This result can easily be verified. From Eq. (16) it can be inferred that the weak formulation is equivalent to solving the eigenvalue equation

$$
H_{\phi} \zeta=E \zeta
$$

on $\mathcal{B} \backslash \Gamma$, where $H_{\phi}$ is given in Eq. (3), subject to the interface boundary condition

$$
n_{i}\left(\left[\left(F^{-1} \circ \phi\right)\left(F^{-1} \circ \phi\right)^{T}\right]_{i j} \frac{\partial \zeta_{ \pm}}{\partial x_{j}} \operatorname{det} F\right)_{m 1}=n_{i}\left(\left[\left(F^{-1} \circ \phi\right)\left(F^{-1} \circ \phi\right)^{T}\right]_{i j} \frac{\partial \zeta_{ \pm}}{\partial x_{j}^{\prime}} \operatorname{det} F\right)_{m 2} .
$$

Notice that the interface boundary condition should be satisfied simultaneously for both $\zeta_{+}$and $\zeta_{-}$ in order for the surface integral appearing in (16) to vanish.

The advantage of formulating the problem in strong form with interface boundary conditions [Eq. (17)] is that solution methods no longer are restricted to weak methods. Hence, it is possible to find solutions by any convenient numerical or exact method. This kind of procedure can be used in most situations involving derivatives of discontinuous quantities, e.g., $k \cdot p$ theory.

\section{ONE- AND TWO-DIMENSIONAL EXAMPLES}

\section{A. Sinusoidal potential} by

The one-dimensional sinusoidal potential is chosen as a first example. This potential is given

$$
V_{i d}\left(x^{\prime}\right)=V_{0}+A \cos \left(\frac{2 \pi}{a} x^{\prime}\right)
$$

for $\left.x^{\prime} \in\right]-N a, N a[$ (see Fig. 1). The real number $a$ is the distance between the maximal value of the potential, and $N \in \mathbb{N}$ is a fixed number. In this example the values $V_{0}=0 \mathrm{eV}$ and $A=5 \mathrm{eV}$ are chosen. In addition, periodic boundary conditions are used. Assuming that the whole potential is deformed when subjected to some deformation $\phi$, the deformed potential is given by 

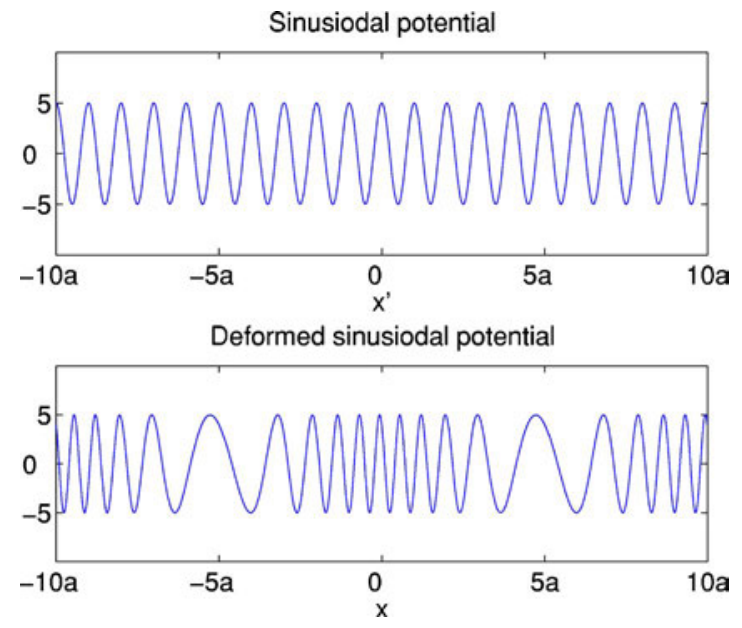

FIG. 1. (Color online) A figure showing the undeformed and deformed sinusoidal potential for $N=10$ and $\epsilon=0.1$.

$$
V_{\phi}(x)=V_{i d}\left(\phi^{-1}(x)\right)=V_{0}+A \cos \left(\frac{2 \pi}{a} \phi^{-1}(x)\right) .
$$

To get an idea of the influence of the deformation on the energies, the deformation is chosen such that

$$
\phi^{-1}(x)=x+\epsilon a \sin \left(\frac{2 \pi}{10 a} x\right)
$$

where it is assumed that $N / 10 \in \mathbb{N}$ and $\epsilon \in \mathbb{R}$ is chosen such that $\partial \phi^{-1} / \partial x>0$ for all $x \in[-N a, N a]$. In Fig. 1 the deformed potential is shown for $N=10$ and $\epsilon=0.1$. The inverse is specified because this insures that it is possible to solve the exact eigenvalue equation and, as a consequence, the first- and second-order approximations can be compared against the exact solution.

This example can be extended to a three-dimensional example by assuming that there is translational symmetry in the $y^{\prime}$ and $z^{\prime}$ directions, and that the system is only deformed in the $x^{\prime}$ direction. The undeformed region can, in this case, be chosen as $\mathcal{B}=]-N a, N a[\times]-N a, N a[\times]$ $-N a, N a[$ with periodic boundary conditions. The potential can then be viewed as originating from a periodic array of plates perpendicular to the $x^{\prime}$ direction. The number $a$ is, in this case, the period of the structure. Ignoring spin, the deformed Hamiltonian is given by

$$
H_{\phi}=-\frac{\hbar^{2}}{2 m} \Delta+V_{\phi}
$$

According to Eq. (8), the second-order Taylor series expansion is

$$
H_{\phi} \approx H_{i d}+D^{(1)}(\phi-i d)+D^{(2)}(\phi-i d, \phi-i d) .
$$

Because of the translational symmetry, the solutions to the eigenvalue problem [Eq. (1)] can always be chosen such that

$$
\widetilde{\psi}\left(x^{\prime}, y^{\prime}, z^{\prime}\right)=\psi\left(x^{\prime}\right) e^{i k_{y^{\prime}} y^{\prime}} e^{i k_{z^{\prime}} z^{\prime}} .
$$

Solving at the zone center, i.e., for $k_{y^{\prime}}=0$ and $k_{z^{\prime}}=0$, it is found that Eq. (8) takes the form 


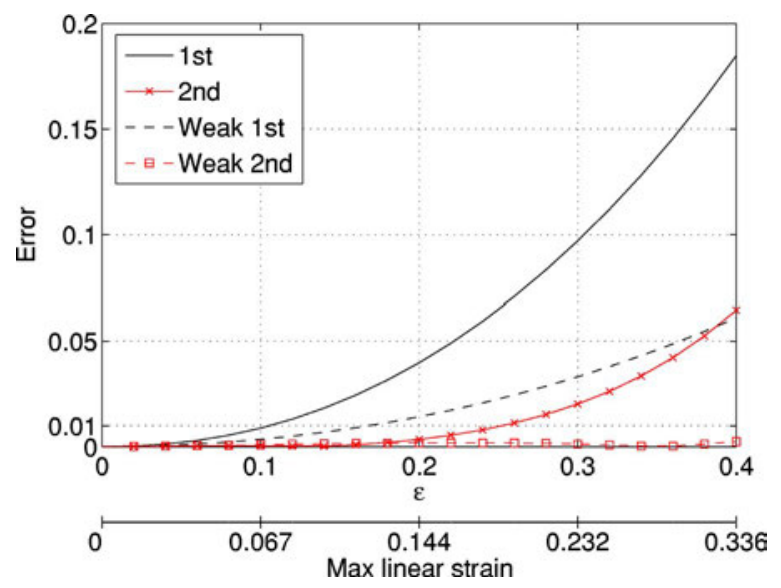

FIG. 2. (Color online) Graph showing the error in the energies for the sinusoidal potential when using the first- and second-order approximations.

$$
H_{\phi} \approx-\frac{\hbar^{2}}{2 m}\left\{\left(1-2\left(\frac{\partial \phi}{\partial x^{\prime}}-1\right)+3\left(\frac{\partial \phi}{\partial x^{\prime}}-1\right)^{2}\right) \frac{\partial^{2}}{\partial x^{\prime 2}}+\left(-\frac{\partial^{2} \phi}{\partial x^{\prime 2}}+3\left(\frac{\partial \phi}{\partial x^{\prime}}-1\right) \frac{\partial^{2} \phi}{\partial x^{\prime 2}}\right) \frac{\partial}{\partial x^{\prime}}\right\}+V_{i d}\left(x^{\prime}\right) \text {. }
$$

The reason why the Taylor series expansion of the potential is exact is that $V_{\phi}(x)=V_{i d}\left(\phi^{-1}(x)\right)$ $=V_{i d}\left(x^{\prime}\right)$. Another way of arriving at Eq. (19) is to derive a theory similar to the one in this article just for the one-dimensional case.

The eigenvalue equation has been solved numerically using FEMLAB, i.e., using a finiteelement method, both for the first- and second-order approximation of the deformed Hamiltonian with respect to $\left(\left(\partial \phi / \partial x^{\prime}\right)-1\right)$. The exact problem, i.e., without making the Taylor series expansion, was also solved using FEMLAB. In Fig. 2 a plot of the error in using the first- and secondorder approximation as a function of $\epsilon$ and the maximum of the linear strain is presented (the solid lines), where the linear strain is given by $\left(\left(\partial \phi / \partial x^{\prime}\right)-1\right)$. The errors of the first- and the secondorder Hamiltonians are given by

$$
\text { Error }^{x}=\left|\frac{E_{1}^{x}-E_{1}^{\text {Exact }}}{E_{1}^{x}}\right|,
$$

$x=1 \mathrm{st}, 2 \mathrm{nd}$, respectively, where $E_{1}^{\text {Exact }}, E_{1}^{1 \mathrm{st}}$, and $E_{1}^{\text {2nd }}$ are the energy of the ground state calculated with the exact, first-order, and second-order Hamiltonians, respectively. From this graph it can be seen that the second-order approximation (crossed red line) is considerably more accurate then the first-order approximation (black line). The first-order approximation has an error of less than $1 \%$ for a max linear strain of up to $6.7 \%$, whereas the second-order approximation is accurate to with in $1 \%$ up to a max linear strain of $18.6 \%$.

Even though it is not necessary to use the weak formulation for the problem in this example (because the deformation is smooth over the whole domain), it is still interesting to see how accurate the results are using the weak approach. First of all, it should be noted that the exact results obtained with the weak formulation are identical to the exact results obtained with the strong formulation. This should also be the case because the formulations are equivalent when everything is smooth. Which formulation is used does however matter when the Taylor series expansion is made. In the weak formulation the Jacobian is present as a consequence of the coordinate transformation of the integral, and it is not present in the strong formulation. So, it is actually the influence of the Jacobian that is under investigation in the following.

According to Sec. II C, the second-order Taylor series expansion of the weak eigenvalues problem has the following form disregarding spin: Find $\tilde{\psi}$ and $E$ such that 


$$
W(\phi)(\eta, \widetilde{\psi}, E)=0,
$$

for all $\eta \in C_{c}^{\infty}(\mathcal{B}, \mathrm{C})$, where

$$
\begin{aligned}
W(\phi)(\eta, \widetilde{\psi}, E) \approx & \frac{\hbar^{2}}{2 m} \int_{-N a}^{N a}\left[\left(1-\left(\frac{\partial \phi}{\partial x^{\prime}}-1\right)+\left(\frac{\partial \phi}{\partial x^{\prime}}-1\right)^{2}\right) \frac{\partial \hat{\eta}^{*}}{\partial x^{\prime}} \frac{\partial \psi}{\partial x^{\prime}}\right] \mathrm{d} x^{\prime} \\
& +\int_{-N a}^{N a}\left[\left(V_{i d}-E\right) \frac{\partial \phi}{\partial x^{\prime}} \hat{\eta}^{*} \psi\right] \mathrm{d} x^{\prime},
\end{aligned}
$$

and

$$
\hat{\eta}=\int_{-N a}^{N a} \int_{-N a}^{N a} \eta \mathrm{d} y^{\prime} \mathrm{d} z^{\prime} .
$$

The first- and second-order approximation of the eigenvalue equation (19) with respect to $\left(\left(\partial \phi / \partial x^{\prime}\right)-1\right)$ is again solved using FEMLAB. The dashed lines in Fig. 2 show the error introduced by using the first- and second-order approximation (black and boxed red lines, respectively). From this figure, it is seen that the second-order approximation is again substantially better than the first-order approximation. In this case, the first-order approximation has an error of less then $1 \%$ for a max linear strain of up to $12 \%$, and the second-order approximation is within an error of $1 \%$ in the whole interval shown, i.e., at least up to a max linear strain of 33.6\%. It is also seen that the first- and second-order approximations of the weak problem are considerably more precise than the first- and second-order approximations of the strong problem. The reason for this goes back to the presence of the Jacobian in the weak formulation in the following way.

First, observe that $\partial \phi^{-1} / \partial x \circ \phi=1 /\left(\partial \phi / \partial x^{\prime}\right)$ and $\operatorname{det}\left(\nabla^{\prime} \phi\right)=\partial \phi / \partial x^{\prime}$. From this it is seen that $\partial \phi^{-1} / \partial x^{\circ} \phi \operatorname{det}\left(\nabla^{\prime} \phi\right)=1$. Because of this, Eq. (14) reduces to

$$
W(\phi)(\eta, \tilde{\psi}, E)=\frac{\hbar^{2}}{2 m} \int_{-N a}^{N a}\left[\frac{\partial \phi^{-1}}{\partial x} \circ \phi \frac{\partial \hat{\eta}^{*}}{\partial x} \frac{\partial \psi}{\partial x^{\prime}}+\left(V_{i d}-E\right) \frac{\partial \phi}{\partial x^{\prime}} \hat{\eta}^{*} \psi\right] \mathrm{d} x^{\prime} .
$$

The reason why the Taylor approximations of this equation is more precise than the Taylor approximations of the strong problem is simply that the first- and second-order Taylor series expansion of $\partial \phi^{-1} / \partial x^{\circ} \phi=1 /\left(\partial \phi / \partial x^{\prime}\right)$ is more accurate than the first- and second-order Taylor series expansion of $\left(\left(\partial \phi^{-1} / \partial x\right) \circ \phi\right)^{2}=\left(1 /\left(\partial \phi / \partial x^{\prime}\right)\right)^{2}$. Hence, the reason why the approximations of the weak formulation are more accurate than the approximations of the strong formulation is that this example is, in essence, a one-dimensional problem. This will be confirmed in the quantumwire example analyzed later.

\section{B. Quantum well}

In this section the influence of deformations on the quantum-well problem is investigated. This is done in order to give an example of a situation where the weak formulation is needed. The quantum-well problem is the one-dimensional problem where the undeformed potential is given by the step function

$$
V_{i d}\left(x^{\prime}\right)=\left\{\begin{array}{ll}
V_{0}, & \left.x^{\prime} \in\right]-d,-a[ \\
V_{1}, & x^{\prime} \in[-a, a] \\
V_{0}, & \left.x^{\prime} \in\right] a, d[
\end{array} .\right.
$$

To simplify matters, spin is again neglected. In this example the following values are used: $V_{0}$ $=5 \mathrm{eV}, V_{1}=0 \mathrm{eV}, a=5 \mathrm{~nm}$, and $d=25 \mathrm{~nm}$, and Dirichlet boundary conditions are imposed. Again, it is assumed that the whole potential is deformed when it is subjected to a deformation $\phi$, i.e., $V_{\phi}(x)=V_{i d}\left(\phi^{-1}(x)\right)$. In nanoscale semiconductor quantum wells, the well material will be de- 


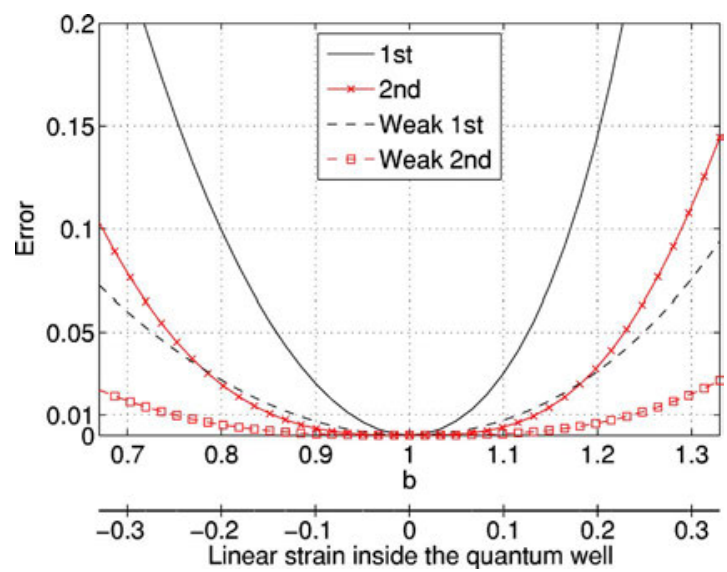

FIG. 3. (Color online) Graph showing the error in the energies for the quantum well when using the first- and second-order approximations.

formed homogeneously, whereas the barrier material will stay undeformed [see, e.g. (18)], i.e., the deformation will have the form

$$
\phi\left(x^{\prime}\right)=\left\{\begin{array}{ll}
x^{\prime}-a(b-1), & \left.x^{\prime} \in\right]-d,-a[ \\
b x^{\prime}, & x^{\prime} \in[-a, a] \\
x^{\prime}+a(b-1), & \left.x^{\prime} \in\right] a, d[
\end{array},\right.
$$

for some $b>0$. The deformation is not differentiable at the interfaces and, as a consequence, the weak formulation is needed. Proceeding as in the sinusoidal example above, it is found that the second-order Taylor series expansion of the weak eigenvalue problem takes the form given in Eq. (19) and the equations that follow.

The first- and second-order approximations have again been solved using FEMLAB. The inverse of the deformation is easily found and, as a consequence, it is also possible to solve the exact weak eigenvalue problem, i.e., without making a Taylor series expansion. The error in the energy of the ground state in using the first- and second-order approximation is shown in Fig. 3 (dashed black and boxed red lines). Here, it is seen that the error introduced by using the first-order approximation is within $1 \%$ for a linear strain of up to $11.6 \%$, whereas the error introduced by using the second-order approximation lies within $1 \%$ for a linear strain of up to $23.8 \%$. Again, it is seen that the second-order approximation is substantially better than the first-order approximation.

In the sinusoidal example above, it was seen that the approximations of the weak formulation gave considerably more accurate results than the approximations of the strong formulation. In order to investigate whether this is the case for the quantum-well problem also, the strong form of the problem with interface boundary conditions (Sec. II C 1) has been solved, again using FEMLAB. It should be noted that the exact solution to the strong form with interface boundary conditions, i.e., without making a Taylor series expansion, is equal to the exact solution to the weak form. This has to be the case because the two formalisms are equivalent as long as no approximations have been made. The second-order approximation of the strong form is given by Eq. (19) above, and the interface boundary conditions are as follows:

$$
\left(\frac{\partial \phi^{-1}}{\partial x} \circ \phi\left(x_{0}\right) \frac{\partial \psi}{\partial x^{\prime}}\left(x_{0}\right)\right)_{L}=\left(\frac{\partial \phi^{-1}}{\partial x} \circ \phi\left(x_{0}\right) \frac{\partial \psi}{\partial x^{\prime}}\left(x_{0}\right)\right)_{R},
$$

for $x_{0}=-a, a$, where $L(R)$ indicates that the expression in brackets is taken in the limit from the left (right). The second-order Taylor series expansion of the interface boundary conditions takes the form 


$$
\begin{aligned}
((1 & \left.\left.-\left(\frac{\partial \phi}{\partial x^{\prime}}\left(x_{0}\right)-1\right)+\left(\frac{\partial \phi}{\partial x^{\prime}}\left(x_{0}\right)-1\right)^{2}\right) \frac{\partial \psi}{\partial x^{\prime}}\left(x_{0}\right)\right)_{L} \\
& =\left(\left(1-\left(\frac{\partial \phi}{\partial x^{\prime}}\left(x_{0}\right)-1\right)+\left(\frac{\partial \phi}{\partial x^{\prime}}\left(x_{0}\right)-1\right)^{2}\right) \frac{\partial \psi}{\partial x^{\prime}}\left(x_{0}\right)\right)_{R},
\end{aligned}
$$

for $x_{0}=-a, a$.

The solid black and crossed red lines in Fig. 3 show the error introduced by using the firstand second-order approximation of the strong form with interface boundary conditions, denoted SForm-first and SForm-second, respectively. Here, we see that the error in using SForm-first is less than $1 \%$ for a linear strain of up to $5.9 \%$, whereas the error introduced using SForm-second is within $1 \%$ for a linear strain of up to $13.5 \%$. So again, it is seen that the second-order approximation is more accurate than the first-order approximation. In addition, it can be seen that the approximations of the weak form are substantially more accurate than the approximations of the strong form with interface boundary conditions. This is expected because this is also a onedimensional problem, so the same reasoning as in the sinusoidal example can be applied.

\section{Quantum wire}

In this section a square-shaped, two-dimensional quantum-wire problem with infinite barrier is investigated. This is done in order to see whether or not the approximations of the weak problem are more accurate than the approximations of the strong form for a two-dimensional problem.

The undeformed two-dimensional domain under investigation is given by $]-5,5[\times]-5,5[$ (in nanometers) and the potential is zero. Dirichlet boundary conditions must be applied because of the infinite barrier. To simplify matters, the following homogeneous deformation is chosen:

$$
\phi\left(\dot{x}^{\prime}, y^{\prime}\right)=\left(\begin{array}{cc}
b & b-1 \\
0 & b
\end{array}\right)\left(\begin{array}{l}
x^{\prime} \\
y^{\prime}
\end{array}\right),
$$

where $b \neq 0$ (this ensures that the Jacobian determinant is positive). The second-order Taylor series expansion of the Strong formulation of the eigenvalue equation takes the form: Find $\psi$ and $E$ such that

$$
H_{\phi} \psi=E \psi
$$

where

$$
\begin{aligned}
H_{\phi} \approx & -\frac{\hbar^{2}}{2 m}\left(\frac{\partial^{2} \psi}{\partial x^{\prime} \partial x^{\prime}}+\frac{\partial^{2} \psi}{\partial y^{\prime} \partial y^{\prime}}-2(b-1)\left(\frac{\partial^{2} \psi}{\partial x^{\prime} \partial x^{\prime}}+\frac{\partial^{2} \psi}{\partial y^{\prime} \partial y^{\prime}}+\frac{\partial^{2} \psi}{\partial x^{\prime} \partial y^{\prime}}\right)\right. \\
& \left.+(b-1)^{2}\left(4 \frac{\partial^{2} \psi}{\partial x^{\prime} \partial x^{\prime}}+3 \frac{\partial^{2} \psi}{\partial y^{\prime} \partial y^{\prime}}+6 \frac{\partial^{2} \psi}{\partial x^{\prime} \partial y^{\prime}}\right)\right) .
\end{aligned}
$$

This can either be derived in a similar manner to the three-dimensional case, or the quantum-wire problem can be extended to a three-dimensional problem by assuming translational symmetry in the third direction. If it is then solved at the zone center in the third direction, the second-order approximation of the deformed Hamiltonian will have the above form. In the latter case the problem is actually closer to a real problem, although a idealized version, because such quantumwire structures can be grown in reality [see Ref. 18].

The solid lines in Fig. 4 show the error resulting from using the first- and second-order approximations (the black and crossed red lines, respectively). Here, it is seen that the first-order approximation is within an error of $1 \%$ for $b-1$ between -0.058 and 0.055 , whereas the energies of the second-order approximation are within an error of $1 \%$ for $b-1$ between -0.197 and 0.098 . Again, it can be seen that the second-order approximation gives substantially better results.

The second-order Taylor series expansion of the weak formulation of the problem takes the form: Find $\psi$ and $E$ such that 


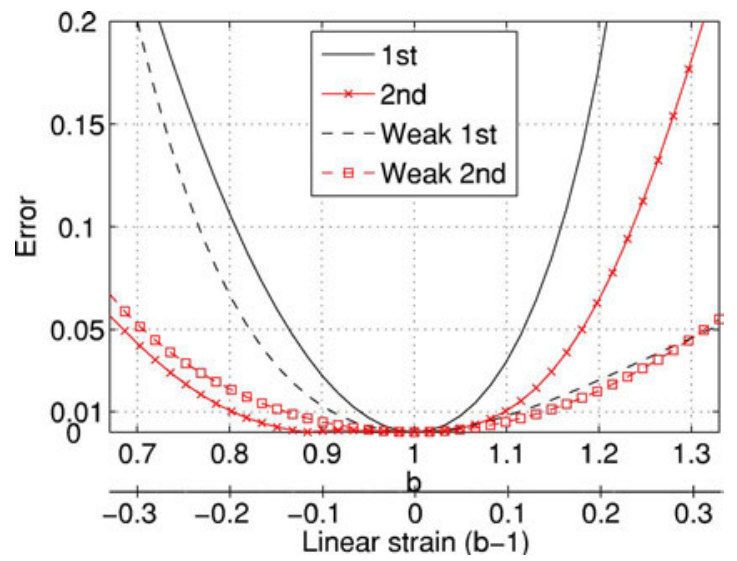

FIG. 4. (Color online) Graph showing the error in the energies for the quantum wire when using the first- and second-order approximations.

$$
W(\phi)(\eta, \psi, E)=0,
$$

for all $\eta \in C_{c}^{\infty}(]-5,5[\times]-5,5[, \mathrm{C})$, where

$$
\begin{aligned}
W(\phi)(\eta, \tilde{\psi}, E) \approx & \int_{-5}^{5} \int_{-5}^{5}\left\{\frac { \hbar ^ { 2 } } { 2 m } \left[\frac{\partial \eta^{*}}{\partial x^{\prime}} \frac{\partial \psi}{\partial x^{\prime}}+\frac{\partial \eta^{*}}{\partial x^{\prime}} \frac{\partial \psi}{\partial x^{\prime}}+\left(-(b-1)+(b-1)^{2}\right)\left(\frac{\partial \eta^{*}}{\partial x^{\prime}} \frac{\partial \psi}{\partial y^{\prime}}+\frac{\partial \eta^{*}}{\partial y^{\prime}} \frac{\partial \psi}{\partial x^{\prime}}\right)\right.\right. \\
& \left.\left.+(b-1)^{2} \frac{\partial \eta^{*}}{\partial x^{\prime}} \frac{\partial \psi}{\partial x^{\prime}}\right]+\left(V_{i d}-E\right)\left(1+2(b-1)+(b-1)^{2}\right) \hat{\eta}^{*} \psi\right\} \mathrm{d} x^{\prime} \mathrm{d} y^{\prime} .
\end{aligned}
$$

From this, the energies can be calculated with respect to a first- and a second-order approximation of the weak form. The dashed lines in Fig. 4 show the error in the energy introduced by using the first- and the second-order approximation of the weak form as a function of both $b$ and $b-1$ (black and boxed red lines, respectively). Here, it is seen that the first-order approximation of the weak form has an error of less than $1 \%$ for $b-1$ between -0.089 and 0.114 , whereas the error of the second-order approximation of the weak form is within an error of $1 \%$ for $b-1$ between -0.139 and 0.14. It is seen, as expected, that the second-order approximation of the weak form is much better than the first-order approximation of the weak form. But, when the approximations of the weak form are compared to the approximations of the strong form, it is seen that the second-order approximation of the strong form is actually more accurate than the second-order approximation of the weak form in the negative direction, whereas it is the second-order approximation of the weak form which is more accurate in the positive direction. Hence, for this two-dimensional problem it is not easy to say which of the two formulations gives the best results. This differs from the observations made in the one-dimensional examples above, where it was seen that the approximations of the weak formulation always were more accurate than the approximations of the strong formulation.

\section{CONCLUSION}

In this article a method to incorporate deformation effects in Hamiltonians has been presented based on a Taylor series expansion with respect to the deformation. This method was exemplified with a Hamiltonian of an inhomogeneous crystal structure including spin. For this Hamiltonian, the second-order Taylor series expansion of the eigenvalue problem was found both in a strong and a weak formulation. The weak formulation was needed in order to be able to handle deformations that might not be differentiable at interfaces. It was also shown that the weak formulation could be transformed into a strong formulation with interface boundary conditions. 
One- and two-dimensional examples were presented, where it was shown that the Taylor series approximations give quite accurate results. In actual fact, an error in the energy within $1 \%$ for a linear strain of up to $5.5 \%$ for the first-order approximations and $9.8 \%$ for the second-order approximations was obtained. For the one-dimensional examples, it was also seen that the approximations of the weak formulation gave considerably more accurate results than the approximations of the strong formulation. But, for the two-dimensional example, this was not the case.

\section{ACKNOWLEDGMENTS}

B. L. would like to thank Jens Gravesen for helpful discussions. L.C.L.Y.V. was supported by an NSF CAREER award (NSF Grant No. 0454849), and by a Research Initiative Grant from Ohio Board of Regents and Wright State University.

\section{APPENDIX A: TAYLOR EXPANSION (STRONG FORMULATION)}

First, it should be noted that all the functions under investigation in this appendix, except the potential $V_{\phi}$, are Frechet differentiable. As a consequence, all the usual rules normally associated with derivatives can be applied.

In the course of finding the Gâteaux derivative of $\hat{H}$, the following lemma will be used:

Lemma A.1. Let $F: \mathcal{D} \rightarrow C(\mathcal{B}, M(3, \mathbb{R}))$ be given by $F(\phi)=\nabla^{\prime} \phi, G \in C(\mathcal{B}, M(3, \mathbb{R}))$, and $\chi$ $\in \hat{C}^{2}$, where $M(3, \mathrm{R})$ is the set of $3 \times 3$ matrices with real coefficients; then

$$
\begin{gathered}
D F(\phi) \cdot \chi=\nabla^{\prime} \chi, \\
D F^{-1}(I) \cdot G=-G,
\end{gathered}
$$

$$
D \operatorname{det} F(I) \cdot G=\operatorname{Tr}(G)
$$

where $I \in C(\mathcal{B}, G L(3, \mathbb{R}))$ is given by $I\left(\mathbf{r}^{\prime}\right)_{i j}=\delta_{i j}, \quad F^{-1}$ is the mapping $F \mapsto F^{-1}$ for $F$ $\in C(\mathcal{B}, G L(3, \mathbb{R}))$, and $\operatorname{det} F$ is the mapping $F \mapsto \operatorname{det} F$. In addition

$$
D^{2} F^{-1}(I) \cdot(G, G)=2(G)^{2}
$$

and

$$
D^{2} \operatorname{det} F(I) \cdot(G, G)=2 \operatorname{Tr}\left([G]_{c o}\right)=2\left(G_{11} G_{22}-G_{12} G_{21}+G_{11} G_{33}-G_{13} G_{31}+G_{22} G_{33}-G_{23} G_{32}\right) \text {, }
$$

where $[G]_{c o}$ is the matrix of cofactors of $G$.

Proof of Lemma A.1. Using Eq. (6), it is seen that

$$
D F(\phi) \cdot \chi=\left.\frac{d}{d t}\left\{\nabla^{\prime}(\phi+t \chi)\right\}\right|_{t=0}=\nabla^{\prime} \chi
$$

Let $F_{0}, G \in C(\mathcal{B}, M(3, \mathbb{R})), F_{0}$ invertible, and let $\alpha \in \mathbb{R}$ be given such that $F_{0}+t G$ is invertible for all $t \in(-\alpha, \alpha)$. Observe that

$$
\frac{\left(F_{0}-t G\right)^{-1}-F_{0}^{-1}}{t}=\frac{F_{0}^{-1}}{t}\left(\left(I+t G F_{0}^{-1}\right)^{-1}-I\right)=-F_{0}^{-1}\left(I+t G F_{0}^{-1}\right)^{-1} G F_{0}^{-1} .
$$

From this it follows that the limit for $t \rightarrow 0$ exists and

$$
D F^{-1}\left(F_{0}\right) \cdot G=-F_{0}^{-1} G F_{0}^{-1} .
$$

With $F_{0}=I$ the second result in Lemma A.1 is found. From Eq. (A1) it is also found that 


$$
D^{2} F^{-1}\left(F_{0}\right) \cdot(G, G)=D\left(-F^{-1} G F^{-1}\right)\left(F_{0}\right) \cdot(G)=2 F_{0}^{-1} G F_{0}^{-1} G F_{0}^{-1} .
$$

With $F_{0}=I$, the third result is obtained.

It is known that

$$
\operatorname{det} F=F_{1} \times F_{2} \cdot F_{3},
$$

where $F_{i}$ is the $i$ th column of $F$. From Eq. (A2), it is seen that

$$
D \operatorname{det} F\left(F_{0}\right) \cdot G=G_{1} \times\left[F_{0}\right]_{2} \cdot\left[F_{0}\right]_{3}+\left[F_{0}\right]_{1} \times G_{2} \cdot\left[F_{0}\right]_{3}+\left[F_{0}\right]_{1} \times\left[F_{0}\right]_{2} \cdot G_{3},
$$

i.e.,

$$
D \operatorname{det} F(I) \cdot G=\operatorname{Tr} G .
$$

Using Eq. (A3), it is found that

$$
D^{2} \operatorname{det} F\left(F_{0}\right) \cdot G=2\left(G_{1} \times G_{2} \cdot\left[F_{0}\right]_{3}+\left[F_{0}\right]_{1} \times G_{2} \cdot G_{3}+G_{1} \times\left[F_{0}\right]_{2} \cdot G_{3}\right),
$$

giving

$$
D^{2} \operatorname{det} F(I) \cdot G=2 \operatorname{Tr} G_{c o} .
$$

\section{The kinetic part $K(F)$}

The second-order Taylor series expansion is given by

$$
K_{F} \approx K_{I}+D K_{I} \cdot(F-I)+\frac{1}{2} D^{2} K_{I} \cdot(F-I, F-I),
$$

where $K_{F}$ is given in Eq. (4). Using the chain rule, Lemma A.1, and the definition of the Gâteaux derivative, it can be shown that

$D K_{F_{0}} \cdot G=\frac{\hbar^{2}}{2 m}\left\{\left[D F^{-1}\left(F_{0}\right) \cdot G\right]_{j i} \frac{\partial}{\partial x_{j}^{\prime}}\left(\left[F_{0}^{-1} \circ \phi_{0}\right]_{k i} \frac{\partial}{\partial x_{k}^{\prime}}\right)+\left[F_{0}^{-1} \circ \phi_{0}\right]_{j i} \frac{\partial}{\partial x_{j}^{\prime}}\left(\left[D F^{-1}\left(F_{0}\right) \cdot G\right]_{k i} \frac{\partial}{\partial x_{k}^{\prime}}\right)\right\}$,

That is

$$
D K_{I} \cdot G=\frac{\hbar^{2}}{2 m}\left\{\left[G+G^{T}\right]_{j k} \frac{\partial}{\partial x_{j}^{\prime}} \frac{\partial}{\partial x_{k}^{\prime}}+\frac{\partial}{\partial x_{i}^{\prime}}\left([G]_{k i}\right) \frac{\partial}{\partial x_{k}^{\prime}}\right\} .
$$

The second derivative of the kinetic part $K(F)$ can be found from Eq. (A4) using that $D^{2} F\left(\phi_{0}\right) \cdot \chi=0$ for all $\chi$ and $\phi_{0}$, giving

$$
\begin{aligned}
D^{2} K_{I} \cdot(G, G)= & -\frac{\hbar^{2}}{m}\left\{\left[G G^{T}\right]_{j k} \frac{\partial}{\partial x_{j}^{\prime}} \frac{\partial}{\partial x_{k}^{\prime}}+[G]_{j i} \frac{\partial}{\partial x_{j}^{\prime}}\left([G]_{k i}\right) \frac{\partial}{\partial x_{k}^{\prime}}+\left[G^{2}+\left[G^{T}\right]^{2}\right]_{j k} \frac{\partial}{\partial x_{j}^{\prime}} \frac{\partial}{\partial x_{k}^{\prime}}\right. \\
& \left.+\frac{\partial}{\partial x_{i}^{\prime}}\left(\left[G^{2}\right]_{k i}\right) \frac{\partial}{\partial x_{k}^{\prime}}\right\} .
\end{aligned}
$$




\section{The spin-orbit part, $H_{\text {so }}$}

The second-order Taylor series expansion of the spin-orbit part is given by

$$
H_{\phi}^{\mathrm{SO}} \approx H_{i d}^{\mathrm{SO}}+D H_{i d}^{\mathrm{SO}} \cdot(\phi-i d)+\frac{1}{2} D^{2} H_{i d}^{\mathrm{SO}} \cdot(\phi-i d, \phi-i d)
$$

where $H_{\phi}^{\mathrm{SO}}$ is given in Eq. (5). Again using (6), Lemma A.1, and the chain rule, it is straightforward to show that

$$
\begin{aligned}
D H_{\phi_{0}}^{\mathrm{SO}} \cdot \chi= & -i \frac{\hbar^{2}}{4 m^{2} c^{2}}\left\{\left(\left[F_{0}^{-1} \circ \phi_{0}\right]^{T} \nabla^{\prime}\left(D V_{\phi_{0}} \cdot \chi\right)\right) \times\left(\left[F_{0}^{-1} \circ \phi_{0}\right]^{T} \nabla^{\prime}\right) \cdot \sigma\right. \\
& +\left(D\left[F^{-1}\right]^{T}\left(F_{0}\right) \cdot\left(\nabla^{\prime} \chi\right) \nabla^{\prime} V_{\phi_{0}}\right) \times\left(\left[F_{0}^{-1} \circ \phi_{0}\right]^{T} \nabla^{\prime}\right) \cdot \sigma \\
& \left.+\left(\left[F_{0}^{-1} \circ \phi_{0}\right]^{T} \nabla^{\prime} V_{\phi_{0}}\right) \times\left(D\left[F^{-1}\right]^{T}\left(F_{0}\right) \cdot\left(\nabla^{\prime} \chi\right) \nabla^{\prime}\right) \cdot \sigma\right\},
\end{aligned}
$$

where $F_{0}=\nabla^{\prime} \phi_{0}$. That is

$$
D H_{i d}^{\mathrm{SO}} \cdot \chi=-i \frac{\hbar^{2}}{4 m^{2} c^{2}}\left\{\left(\nabla^{\prime}\left(D V_{i d} \cdot \chi\right)\right) \times \nabla^{\prime} \cdot \sigma-\left(\nabla^{\prime} \chi^{T} \nabla^{\prime} V_{i d}\right) \times \nabla^{\prime} \cdot \sigma-\left(\nabla^{\prime} V_{i d}\right) \times \nabla^{\prime} \chi^{T} \nabla^{\prime} \cdot \sigma\right\}
$$

From Eq. (A5), the second derivative of $H_{\mathrm{SO}}$ is found, arriving at

$$
\begin{aligned}
D^{2} H_{i d}^{\mathrm{SO}} \cdot(\chi, \chi)= & -i \frac{\hbar^{2}}{2 m^{2} c^{2}}\left\{-\left(\nabla^{\prime} \chi^{T} \nabla^{\prime}\left(D V_{i d} \cdot \chi\right)\right) \times \nabla^{\prime} \cdot \sigma-\left(\nabla^{\prime}\left(D V_{i d} \cdot \chi\right)\right) \times\left(\nabla^{\prime} \chi^{T} \nabla^{\prime}\right) \cdot \sigma\right. \\
& +\frac{1}{2}\left(\nabla^{\prime}\left(D^{2} V_{i d} \cdot(\chi, \chi)\right)\right) \times\left(\nabla^{\prime}\right) \cdot \sigma+\left(\nabla^{\prime} \chi^{T} \nabla^{\prime} V_{i d}\right) \times\left(\nabla^{\prime} \chi^{T} \nabla^{\prime}\right) \cdot \sigma \\
& \left.+\left(\left[\nabla^{\prime} \chi^{T}\right]^{2} \nabla^{\prime} V_{i d}\right) \times \nabla p^{\prime} \cdot \sigma+\left(\nabla^{\prime} V_{i d}\right) \times\left(\left[\nabla^{\prime} \chi^{T}\right]^{2} \nabla^{\prime}\right) \cdot \sigma\right\} .
\end{aligned}
$$

\section{APPENDIX B: TAYLOR EXPANSION (WEAK FORMULATION)}

In the course of determining the weak formulation, Theorem B.1 was used. First, two problems are introduced.

(I) Find $\zeta$ and $E$ such that

$$
W(\phi)(\xi \circ \phi, \zeta, E)=0,
$$

for all $\xi_{+}, \xi_{-} \in C_{c}^{\infty}(\phi(\mathcal{B}), C)$.

(II) Find $\zeta$ and $E$ such that

$$
W(\phi)(\eta, \zeta, E)=0
$$

for all $\eta_{+}, \eta_{-} \in C_{c}^{\infty}(\mathcal{B}, \mathrm{C})$.

Theorem B.1. Problem I and II are equivalent.

Proof. Let $\zeta$ and $E$ be a solution to problem (II), and assume that there exists a $\xi=\left(\xi_{+}, \xi_{-}\right)^{T}$, with $\xi_{+}, \xi_{-} \in C_{c}^{\infty}(\phi(\mathcal{B}), \mathrm{C})$, such that $W(\phi)\left(\xi^{\circ} \phi, \zeta, E\right) \neq 0$. It is easily seen, using a regularizer (see Ref. 19), that there exists two sequences of functions in $C_{c}^{\infty}(\mathcal{B}, C)$, denoted $\xi_{+\epsilon}$ and $\xi_{-\epsilon}$, such that $\left(\xi_{+\epsilon}, \xi_{-\epsilon}\right)^{T} \rightarrow \xi^{\circ} \phi$ for $\epsilon \rightarrow 0$. Using the dominated convergence theorem (see Ref. 16), it can be shown that

$$
\lim _{\epsilon \rightarrow 0}\left(W(\phi)\left(\left(\xi_{+\epsilon}, \xi_{-\epsilon}\right)^{T}, \zeta, E\right)\right)=W(\phi)\left(\xi^{\circ} \phi, \zeta, E\right) ;
$$

at the same time using that $\zeta$ and $E$ is a solution to problem (II), it is found that 


$$
\lim _{\epsilon \rightarrow 0}\left(W(\phi)\left(\left(\xi_{+\epsilon}, \xi_{-\epsilon}\right)^{T}, \zeta, E\right)\right)=0 .
$$

But, this contradicts the assumption that $W(\phi)(\xi \circ \phi, \zeta, E) \neq 0$. From this it is seen that $W(\phi)\left(\xi^{\circ} \phi, \zeta, E\right)=0$ for all $\xi_{+}, \xi_{-} \in C_{c}^{\infty}(\phi(\mathcal{B}), C)$, showing that $\zeta$ and $E$ is also a solution to problem (I).

That a solution to problem (I) is also a solution to problem (II) is shown in a similar manner by changing to coordinates on $\phi(\mathcal{B})$ in the integrals.

\section{The kinetic part, $K_{\phi}^{ \pm}$}

Using the expressions in Lemma A.1, it is found that

$$
\begin{aligned}
{\left[D K_{\phi_{0}}^{ \pm} \cdot \chi\right]\left(\eta_{ \pm}, \zeta\right)=} & \frac{\hbar^{2}}{2 m} \int_{\mathcal{B} \Gamma \Gamma}\left\{\left[\left(D F^{-1}\left(F_{0}\right) \cdot \nabla^{\prime} \chi\right)\left(F_{0}^{-1} \circ \phi\right)^{T}\right]_{i j} \frac{\partial\left(\eta_{ \pm}\right)^{*}}{\partial x_{i}^{\prime}} \frac{\partial \zeta_{ \pm}}{\partial x_{j}^{\prime}} \operatorname{det} F_{0}\right. \\
& +\left[\left(F_{0}^{-1} \circ \phi\right)\left(D F^{-1}\left(F_{0}\right) \cdot \nabla^{\prime} \chi\right)^{T}\right]_{i j} \frac{\partial\left(\eta_{ \pm}\right)^{*}}{\partial x_{i}^{\prime}} \frac{\partial \zeta_{ \pm}}{\partial x_{j}^{\prime}} \operatorname{det} F_{0} \mathrm{~d} V^{\prime} \\
& \left.+\left[\left(F_{0}^{-1} \circ \phi\right)\left(F_{0}^{-1} \circ \phi\right)^{T}\right]_{i j} \frac{\partial\left(\eta_{ \pm}\right)^{*}}{\partial x_{i}^{\prime}} \frac{\partial \zeta_{ \pm}}{\partial x_{j}^{\prime}} D \operatorname{det} F\left(F_{0}\right) \cdot \nabla^{\prime} \chi\right\} \mathrm{d} V^{\prime},
\end{aligned}
$$

where $F_{0}=\nabla^{\prime} \phi_{0}$, giving

$$
\left[D K_{i d}^{ \pm} \cdot \chi\right]\left(\eta_{ \pm}, \zeta\right)=-\frac{\hbar^{2}}{2 m} \int_{\mathcal{B} \Gamma \Gamma}\left[\nabla^{\prime} \chi+\nabla^{\prime} \chi^{T}\right]_{i j} \frac{\partial\left(\eta_{ \pm}\right)^{*}}{\partial x_{i}^{\prime}} \frac{\partial \zeta_{ \pm}}{\partial x_{j}^{\prime}}-\frac{\partial\left(\eta_{ \pm}\right)^{*}}{\partial x_{i}^{\prime}} \frac{\partial \zeta_{ \pm}}{\partial x_{i}^{\prime}} \operatorname{Tr}\left(\nabla^{\prime} \chi\right) \mathrm{d} V^{\prime}
$$

Continuing from Eq. (B1), it is seen that

$$
\begin{aligned}
{\left[D^{2} K_{i d}^{ \pm} \cdot(\chi, \chi)\right]\left(\eta_{ \pm}, \zeta\right)=} & \frac{\hbar^{2}}{m} \int_{\mathcal{B} \Gamma \Gamma}\left\{\left[\left(\nabla^{\prime} \chi\right)^{2}+\left(\nabla^{\prime} \chi^{T}\right)^{2}+\left(\nabla^{\prime} \chi\right)\left(\nabla^{\prime} \chi\right)^{T}\right]_{i j} \frac{\partial\left(\eta_{ \pm}\right)^{*}}{\partial x_{i}^{\prime}} \frac{\partial \zeta_{ \pm}}{\partial x_{j}^{\prime}} \mathrm{d} V^{\prime}\right. \\
& \left.-\left[\nabla^{\prime} \chi+\nabla^{\prime} \chi \chi^{T}\right]_{i j} \frac{\partial\left(\eta_{ \pm}\right)^{*}}{\partial x_{i}^{\prime}} \frac{\partial \zeta_{ \pm}}{\partial x_{j}^{\prime}} \operatorname{Tr}\left(\nabla^{\prime} \chi\right) \mathrm{d} V^{\prime}+\frac{\partial\left(\eta_{ \pm}\right)^{*}}{\partial x_{i}^{\prime}} \frac{\partial \zeta_{ \pm}}{\partial x_{i}^{\prime}} \operatorname{Tr}\left(\left[\nabla^{\prime} \chi\right]_{c o}\right)\right\} \mathrm{d} V^{\prime}
\end{aligned}
$$

\section{The potential part, $V_{\phi}^{ \pm}$}

Again using Lemma A.1, it is found that

$$
\left[D V_{\phi_{0}}^{ \pm} \cdot \chi\right]\left(\eta_{ \pm}, \zeta\right)=\int_{\mathcal{B}} \eta_{ \pm}^{*}\left(D V_{\phi_{0}} \cdot \chi \operatorname{det} F_{0}+V_{\phi_{0}} \circ \phi_{0} D \operatorname{det} F\left(F_{0}\right) \cdot \nabla^{\prime} \chi\right) \zeta_{ \pm} \mathrm{d} V^{\prime}
$$

that is

$$
\left[D V_{i d}^{ \pm} \cdot \chi\right]\left(\eta_{ \pm}, \zeta\right)=\int_{\mathcal{B}} \eta_{ \pm}^{*}\left(D V_{i d} \cdot \chi+V_{i d} \operatorname{Tr}\left(\nabla^{\prime} \chi\right)\right) \zeta_{ \pm} \mathrm{d} V^{\prime}
$$

The second-order derivative is found using Eq. (B2) and Lemma A.1, giving

$$
\left[D^{2} V_{\phi_{0}}^{ \pm} \cdot \chi\right]\left(\eta_{ \pm}, \zeta\right)=\int_{\mathcal{B}} \eta_{ \pm}^{*}\left(D^{2} V_{i d} \cdot(\chi, \chi)+2 D V_{i d} \cdot \chi \operatorname{Tr}\left(\nabla^{\prime} \chi\right)+2 V_{i d} \operatorname{Tr}\left(\left[\nabla^{\prime} \chi\right]_{c o}\right)\right) \zeta_{ \pm} \mathrm{d} V^{\prime}
$$




\section{The spin-orbit part, $H_{\phi}^{\mathrm{SO}, \pm}$}

Finding the derivatives of the spin-orbit part is straightforward, giving

$$
\left[D H_{i d}^{\mathrm{SO}, \pm} \cdot \chi\right]\left(\eta_{ \pm}, \zeta\right)=-i \frac{\hbar^{2}}{2 m^{2} c^{2}} \int_{\mathcal{B} \backslash \Gamma} \eta_{ \pm}^{*}\left(\nabla^{\prime} \chi^{T} \sigma \times \nabla^{\prime} V_{i d} \cdot \nabla^{\prime} \zeta+\sigma \times \nabla^{\prime}\left(D V_{i d} \cdot \chi\right) \cdot \nabla^{\prime} \zeta\right)_{ \pm} \mathrm{d} V^{\prime}
$$

and

$$
\begin{aligned}
{\left[D^{2} H_{i d}^{\mathrm{SO}, \pm} \cdot(\chi, \chi)\right]\left(\eta_{ \pm}, \zeta\right)=} & -i \frac{\hbar^{2}}{2 m^{2} c^{2}} \int_{\mathcal{B} \backslash \Gamma} \eta_{ \pm}^{*}\left(2 \nabla^{\prime} \chi^{T} \sigma \times \nabla^{\prime}\left(D V_{i d} \cdot \chi\right) \cdot \nabla^{\prime} \zeta\right)_{ \pm} \mathrm{d} V^{\prime} \\
& -i \frac{\hbar^{2}}{2 m^{2} c^{2}} \int_{\mathcal{B} \backslash \Gamma} \eta_{ \pm}^{*}\left(\sigma \times \nabla^{\prime}\left(D^{2} V_{i d} \cdot(\chi, \chi)\right) \cdot \nabla^{\prime} \zeta\right)_{ \pm} \mathrm{d} V^{\prime}
\end{aligned}
$$

${ }^{1}$ E. P. O'Reilly, Semicond. Sci. Technol. 4, 121 (1989).

${ }^{2}$ H. T. Johnson and R. Bose, J. Mech. Phys. Solids 51, 2085 (2003).

${ }^{3}$ B. Bimberg, M. Grundmann, and N. N. Ledentsov, Quantum Dot Heterostructures (John Wiley \& Sons, New York, 1999).

${ }^{4}$ G. L. Bir and G. E. Pikus, Symmetry and Strain-induced Effects in Semiconductors (Wiley, New York, 1974).

${ }^{5}$ Y. Zhang, Phys. Rev. B 49, 14352 (1994).

${ }^{6}$ T. Suzuki, Phys. Rev. B 64, 233310 (2001).

${ }^{7}$ V. Aldaya and J. A. de Azárraga, J. Phys. A 13, 2545 (1980).

${ }^{8}$ R. Santroprete, B. Koiller, R. B. Capaz, P. Kratzer, Q. K. K. Lui, and M. Scheffler, Phys. Rev. B 68, 235311 (2003).

${ }^{9}$ J. R. Downes, D. A. Faux, and E. P. O’Reilly, J. Appl. Phys. 81, 6700 (1997).

${ }^{10}$ V. A. Fonoberov and A. A. Balandin, J. Appl. Phys. 94, 7178 (2003).

${ }^{11}$ A. D. Andreev and E. P. O'Reilly, Phys. Rev. B 62, 15851 (2000).

${ }^{12}$ P. D. Hislop and I. M. Sigal, Introduction to Spectral Theory With Applications to Schrödinger Operators (Springer, New York, 1996).

${ }^{13}$ L. M. Graves, Trans. Am. Math. Soc. 29, 193 (1927).

${ }_{15}^{14}$ J. T. Schwartz, Nonlinear Functional Analysis (Gordon and Breach Science Publishers, New York, 1969).

${ }^{15}$ S. J. Gustafson and I. M. Sigal, Mathematical Concepsts of Quantum Mechanics (Springer, Berlin, 2003).

${ }^{16}$ S. Lang, Real and Functional Analysis, 3rd ed. (Springer-Verlag, New York, 1993).

${ }^{17}$ K. Pinardi, U. Jain, S. C. Jain, H. E. Maes, R. Van Overstraeten, and M. Willander, J. Appl. Phys. 83, 4724 (1998).

${ }^{18}$ P. Ils, Ch. Greus, A. Forchel, V. D. Kulakovskii, N. A. Grippius, and S. G. Tikhodeev, Phys. Rev. B 51, 4272 (1995).

${ }^{19}$ W. P. Ziemer, Weakly Differentiable Functions (Springer-Verlag, New York, 1989). 